\title{
The lack of construct validity when assessing clinical clerks during their anesthesia rotations
}

\author{
Amir Hamid, BSc (Hons) (D) Mary Lou Schmuck, BA · Daniel Cordovani, MD, MSc
}

Received: 15 September 2019/Revised: 8 November 2019/Accepted: 9 February 2020/Published online: 24 February 2020

(C) Canadian Anesthesiologists' Society 2020

\section{To the Editor,}

As part of our ongoing program assessment, we analyze the grades that anesthesiologists assign to McMaster University medical students during their core anesthesia clerkship rotation. This assessment has generally revealed grade leniency and poor predictability of success on the Medical Council of Canada Qualifying Examination Part 1 (MCCQE1), leading us to question the validity of this assessment tool.

To further investigate this, we examined the final evaluations from the two-week anesthesia rotation of all 205 undergraduate medical students in the class of 2018 at McMaster University. ${ }^{\mathrm{A}}$ Final evaluations use an average of daily clinical evaluations assessing eight different domains of medical competence on a ten-point Likert Scale. Scores are anchored at 1 ("below expectations") and 10 ("exceptional") with 5 being ("meets expectations"). On average, each student receives nine daily evaluations.

The mean (standard deviation [SD]) final mark was 7.1 (0.6). As scores of 1-4 were never endorsed, no student was shown to fall below the "meets expectations" designation in any domain (Figure) inherently limiting the construct validity of the assessment tool. There was no

\footnotetext{
A. Hamid, BSc (Hons) ( $\square)$

McMaster University Michael G DeGroote School of Medicine, Hamilton, ON, Canada

e-mail: amir.hamid@medportal.ca
}

M. L. Schmuck, BA

Faculty of Health Sciences, McMaster University, Hamilton, ON, Canada

\section{Cordovani, MD, MSc}

Department of Anesthesia, McMaster University Michael G DeGroote School of Medicine, Hamilton, ON, Canada significant difference in the eight domains between pass and fail cohorts of the MCCQE1. A "Red Zone" status (defined as $>2$ SD below the class cohort mean) did not predict a failing MCCQE1 score. A discriminant function analysis (DFA) was used to identify MCCQE1 pass $v s$ fail status. It resembles a regression equation except that the goal is to predict a category rather than the value of a continuous dependent variable. The DFA correctly identified $88.8 \%$ of the MCCQE1 failures, but only $62.4 \%$ of the MCCQE1 passes. Despite the absence of grades $<5$, based on a DFA it appears that it is possible to retrospectively identify students who were weakest compared with their peers based on the daily evaluations. We suspect full use of the scale anchors (even if scores of 5 through 10 appear merited) would allow borderline students to be identified earlier in a more accurate continuum of clinical performance.

"Failure to Fail" is pervasive in medical education. ${ }^{1}$ Despite accurate assessment of a medical trainee's performance, there are still barriers that prevent preceptors from identifying underperforming medical learners. ${ }^{1}$ Faculty development initiatives and support from administration can have benefits, but simpler interventions such as clearer rubrics by which to measure student performance can influence assessors to expand the range of scores they use, which provides more valid, reliable, and predictive assessments. ${ }^{2}$

\footnotetext{
$\bar{A}$ Research Ethics Board Approval: Hamilton Integrated Research Ethics Board (HIREB) operates in compliance with and is constituted in accordance with the requirements of the Tri-Council Policy Statement (TCPS2), noting that Article 2.5 states that quality assurance and quality improvement studies do not fall within the scope of REB review. Considering this Letter to the Editor reports findings of a routine program evaluation, submission to the HIREB was not required.
} 
Figure The average preceptorassigned grades of 205

McMaster class of 2018

students during their anesthesia rotation. Frequency is presented as the number of students achieving a given grade. There were no scores below 5 .

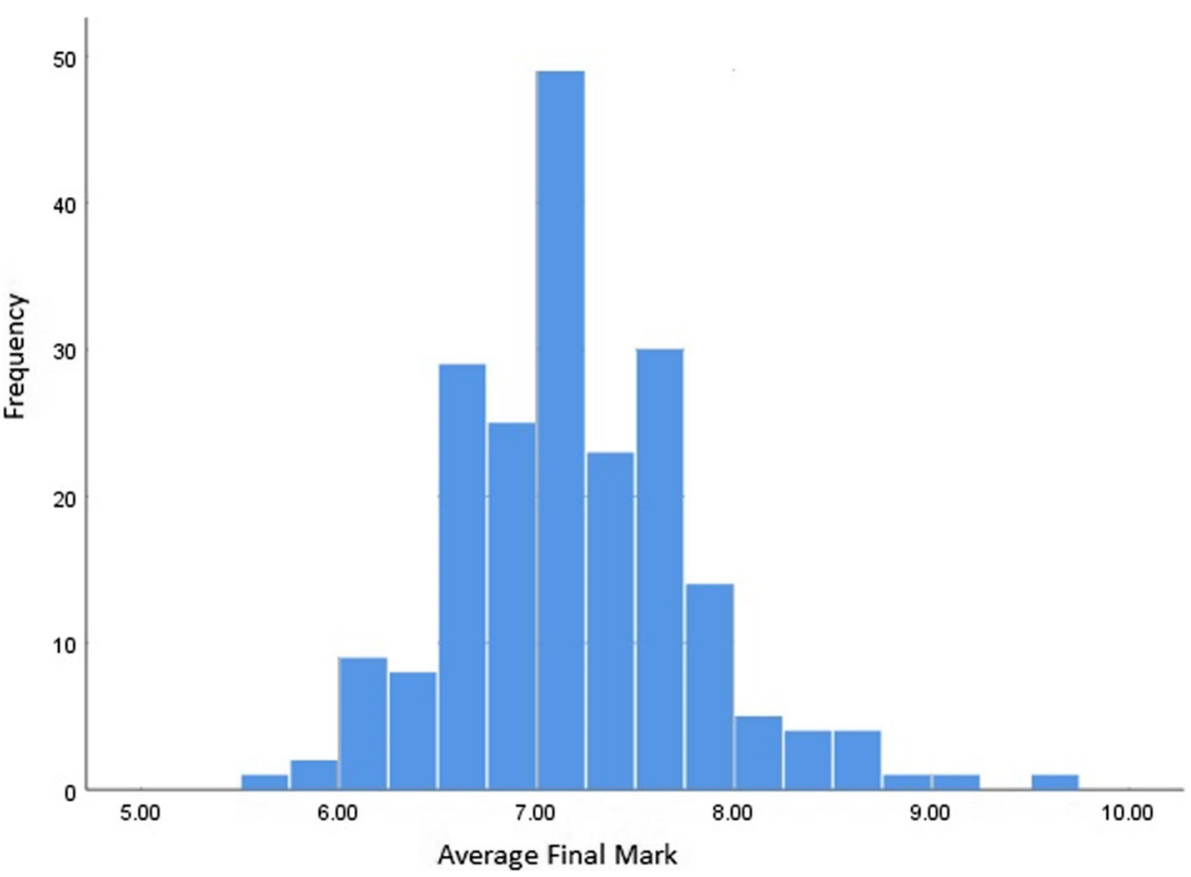

Early identification of students in difficulty who are at risk of failing their licensing examination is relevant to medical educators. ${ }^{3}$ Admittedly, a single comparison with another examination is not a comprehensive validation strategy. Nevertheless, there has been a call for more locally available data related to validation to be disseminated. ${ }^{4}$

Although anesthesia-related content corresponds to a small fraction of what is included in the MCCQE1, several hours of direct observation in the clinical setting done by multiple assessors over a two-week period during clerkship, along with a 1:1 staff to student ratio, should provide reasonable insight into a student's ability and competency as a medical graduate. Our data does not corroborate this statement. In contrast, the Multiple Mini Interview, which corresponds to roughly two hours of an interview process for the selection of medical students, has shown good predictability of MCCQE1 performance. ${ }^{5} \mathrm{We}$ believe one of the contributing factors for the efficacy of the Multiple Mini Interview is that its assessors are trained to use the full range of scores when assessing prospective MD candidates. The value of avoiding a "ceiling effect" by using the full range of scores is necessary to increase the clinical evaluation scale's validity. We encourage other institutions to report their assessment strategies and validation procedures.

\section{Conflicts of interest None.}

Funding statement This data was conducted and analyzed by internal staff with no external funding as part of routine program evaluation at McMaster University.

Editorial responsibility This submission was handled by Dr. Hilary P. Grocott, Editor-in-Chief, Canadian Journal of Anesthesia.

\section{References}

1. Yepes-Rios M, Dudek N, Duboyce R, Curtis J, Allard RJ, Varpio L. The failure to fail underperforming trainees in health professions education: a BEME systematic review: BEME Guide no. 42. Med Teach 2016; 38: 1092-9.

2. Baker $K$. Determining resident clinical performance: getting beyond the noise. Anesthesiology 2011; 115: 862-78.

3. Barber C, Hammond R, Gula L, Tithecott G, Chahine S. In search of black swans: identifying students at risk of failing licensing examinations. Acad Med 2018; 93: 478-85.

4. Cook DA, Brydges R, Ginsburg S, Hatala R. A contemporary approach to validity arguments: a practical guide to Kane's framework. Med Educ 2015; 49: 560-75.

5. Eva KW, Reiter HI, Rosenfeld J, Trinh K, Wood TJ, Norman GR. Association between a medical school admission process using the multiple mini-interview and national licensing examination scores. JAMA 2012; 308: 2233-40.

Publisher's Note Springer Nature remains neutral with regard to jurisdictional claims in published maps and institutional affiliations. 